\title{
Madelung disease
}

\section{Fang-ying Lin MD, Tsung-Lin Yang MD PhD}
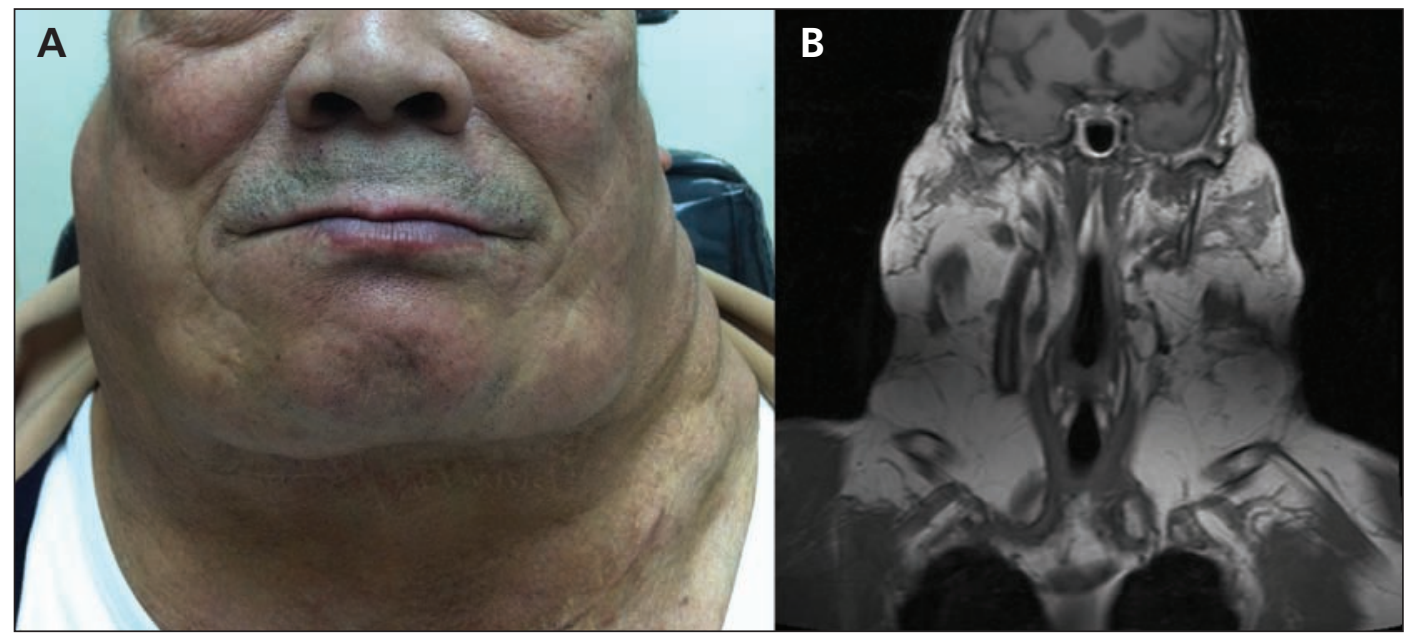

Figure 1: (A) A 68-year-old man with a long-standing history of heavy alcohol use presenting with softtissue swellings over his head and neck. (B) $T_{1}$-weighted magnetic resonance image showing nonencapsulated masses with soft-tissue density widely distributed over the superficial and deep fascial spaces of the head, neck, back and upper trunk.

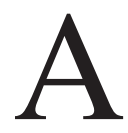

68-year-old man with an extended history of heavy alcohol use presented with soft, painless and slow-growing swellings over his neck, shoulders and head (Figure 1A). Magnetic resonance imaging of his head and neck showed nonencapsulated soft-tissue masses widely distributed over the superficial and deep fascial spaces (Figure 1B). Surgery was scheduled because of disfigurement and limitations of movement. Pathologic examination showed lipomatous hypertrophy, confirming our clinical diagnosis of Madelung disease. Our patient recovered from surgery uneventfully and has had no obvious recurrence for 11 months.

Madelung disease, also known as multiple symmetric lipomatosis, is characterized by benign, nonencapsulated fatty masses around the head and neck, the trunk and the upper extremities. ${ }^{1}$ Its prevalence is about 1:25000 with men affected more frequently than women by a ratio of 20:1. ${ }^{1}$ The vast majority $(90 \%)$ of people with Madelung disease have a history of alcoholism. ${ }^{1}$ Dysphagia and dyspnea may result from laryngeal or mediastinal involvement. The etiology of Madelung disease remains largely unknown. ${ }^{2}$ Most instances are sporadic, but a familial form characterized by maternally inherited mitochondrial gene mutation has been reported. ${ }^{3}$ Madelung disease may be associated with diabetes mellitus, hyperuricemia, hypothyroidism, liver disease or peripheral neuropathy. Its differential diagnosis includes solitary lipoma, encapsulated lipoma, familial multiple lipomatosis and liposarcoma. ${ }^{1}$

Extensive lipectomy remains the standard treatment for Madelung disease. ${ }^{1}$ There is little evidence supporting interventions beyond surgery and dealing with alcohol abuse. ${ }^{1}$ Recurrence is common. Abstinence from alcohol is not associated with spontaneous regression, but it has been reported to reduce the rate of recurrence. ${ }^{4}$

\section{References}

1. González-Garciá R, Rodríguez-Campo FJ, Sastre-Pérez J, et al. Benign symmetric lipomatosis (Madelung's disease): case reports and current management. Aesthetic Plast Surg 2004; 28:108-12, discussion 113 .

2. Nisoli E, Regianini L, Briscini L, et al. Multiple symmetric lipomatosis may be the consequence of defective noradrenergic modulation of proliferation and differentiation of brown fat cells. J Pathol 2002;198:378-87.

3. Gámez J, Playán A, Andreu AL, et al. Familial multiple symmetric lipomatosis associated with the A8344G mutation of mitochondrial DNA. Neurology 1998;51:258-60.

4. Guilemany JM, Romero E, Blanch JL. An aesthetic deformity: Madelung's disease. Acta Otolaryngol 2005;125:328-30.
Competing interests: None declared.

This article has been peer reviewed.

Affiliations: From the Department of

Otolaryngology (Lin, Yang), National Taiwan University Hospital and National Taiwan University College of Medicine; and the Research Center for Developmental Biology and Regenerative Medicine (Yang), National Taiwan University, Taipei, Taiwan

Correspondence to: Tsung-Lin Yang, yangtl@ntu.edu.tw

CMAJ 2013. DOI:10.1503 /cmaj.120191 